\title{
6 Describing the Significance of the Results to Optimize the Structure of the Pressing Chamber
}

In this section, the significance of the conducted experiments and results are discussed as well as their effects on optimizing the construction of the pressing chamber and pressing structure itself. During the experiments and during the evaluation of the experiments, the results are significant regarding the construction of the pressing machines and pressing chambers. This section will briefly describe the significance of the results with respect to the construction of pressing chambers and machines.

\subsection{Effect of Pressing Temperature}

The pressing temperature has the largest impact on the whole pressing process. The pressing temperature affects the plasticization of lignin during the pressing process. Lignin, when in liquid state, can bind better to the pressed material. The effect of the pressing pressure, which is followed by cooling, creates a solid briquette. Based on the experimental results, it can be argued that the optimal pressing temperature, from a quality point of view, is more important than the pressing pressure. From practical experiences, it is also recognized that obtaining the optimal temperature in a pressing machine is cheaper than reaching an optimal value of pressure. It is then logical, when constructing pressing machines, to use a device with ability to regulate temperature. Another effect of the pressing temperature on the process is the experimental observation that, during the pressing process and with the use of the optimal pressing temperature, less force is required for removing the briquette from the pressing chamber when compared to that from pressing with no applied heat in the process. When pressing under higher temperatures, plasticization of the lignin occurs, followed by its subsequent mixing within the material particles, which creates a smooth shiny film on the surface of the briquette. This film causes a smaller friction coefficient between the briquette and chamber wall.

\subsection{Functional Dependence $\rho=f(p, T)$}

Finding this functional dependence is the "alpha and omega" of the whole pressing process for the optimal construction of a pressing machine. Not one manufacturer of these machines can say with certainty what the appropriate pressing pressures for making a quality briquette according to standards are. Too often these pressing machines are oversized and thus economically demanding. Finding the functional dependence can help when constructing a pressing machine, sizing the drive, designing the mechanism, and sizing of the shape and dimensions of the pressing 
chamber, etc. Of course, it is necessary to conduct many more experiments because the results of our experiments are valid only for pine and only for certain intervals of pressing temperature, pressing pressure, material water content, and fraction size. To obtain better knowledge about the behaviour of the pressed material within the densification process, it is necessary to repeatedly perform experiments and supplement the results with respect to other materials. It is important to know that each material type is necessary to experiment with separately. In practice, the demands for optimal parameters are ever-increasing for the densification of varying material mixtures (for example: 30\% pine, 30\% spruce, 30\% fir); therefore, experiments utilizing such mixtures are necessary to perform in order to obtain the optimal parameters for their use.

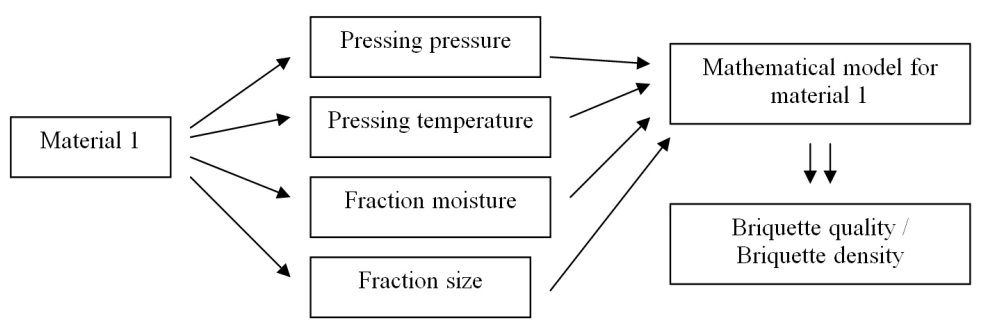

Figure 6.1: Methodology for creating the mathematical model in the first stage of the experiment (Križan, Matúš \& Svátek, 2012; Svátek \& Križan, 2013).

In Fig. 4.3, the briquette density change due to changes in temperature and pressure is shown. If we look closely at the change of briquette density, pressed at $95 \mathrm{MPa}$, then only under the effect of the change of temperature from $85^{\circ} \mathrm{C}$ to $115^{\circ} \mathrm{C}$ the density changes by around $25.8 \%$ from $0.9378 \mathrm{~kg} \cdot \mathrm{dm}^{-3}$ to $1.1797 \mathrm{~kg} . \mathrm{dm}^{-3}$. If we press a material of the same characteristics with a pressure of $159 \mathrm{MPa}$, then, under the effect of temperature from $85^{\circ} \mathrm{C}$ to $115^{\circ} \mathrm{C}$, the density changes by around $15.6 \%$ from $1.0604 \mathrm{~kg} \cdot \mathrm{dm}^{-3}$ to $1.2255 \mathrm{~kg} . \mathrm{dm}^{-3}$. This means that, although we pressed with a $67.4 \%$ higher pressure than in the first case, we achieved very small gains in density at the mentioned temperature values. At a temperature of $85^{\circ} \mathrm{C}$, the change in briquette density is around $13 \%$, while at a temperature of $115^{\circ} \mathrm{C}$, the change in density is no more than $4 \%$. This proves the assumption that pressing at lower pressure and higher temperature is more effective. The following figure shows the effects of interacting pressing pressure and temperature on the density of the pine briquette at various moisture levels. 

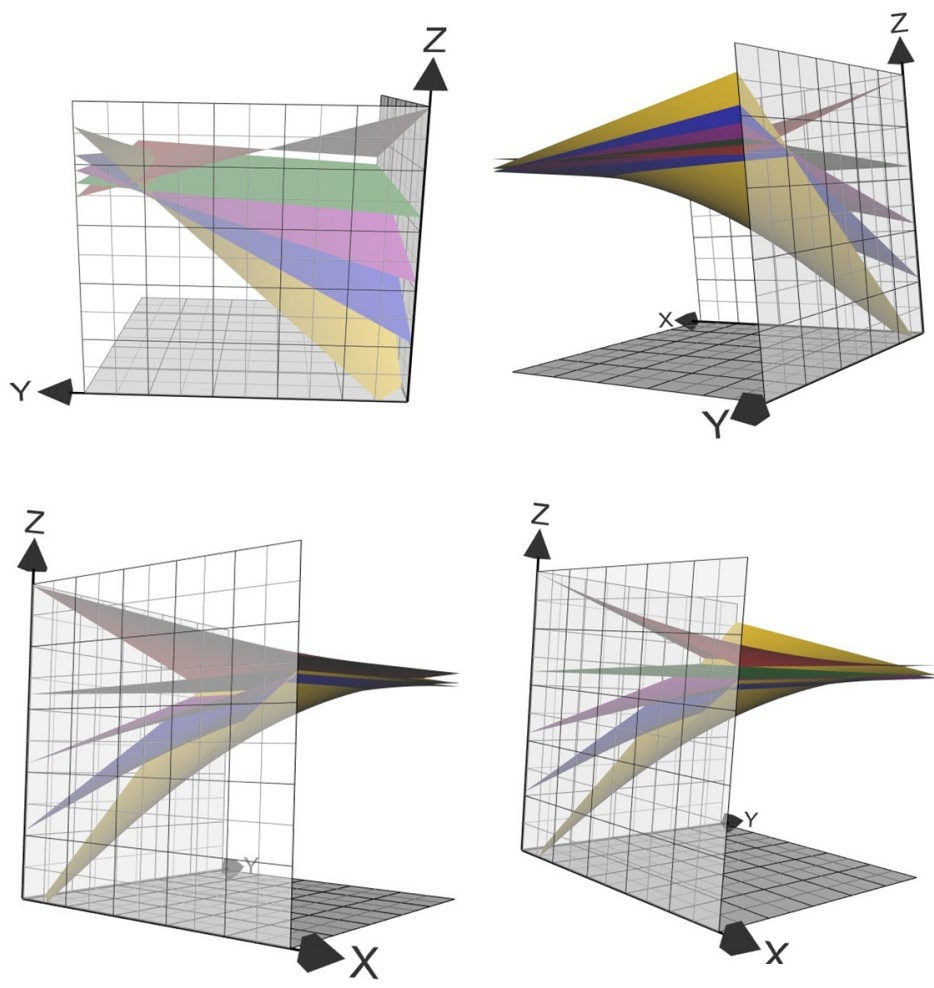

Figure 6.2: Surface response of the density of pine briquettes at a constant fraction size $2 \mathrm{~mm} /$ Labeling: $x$-axis $\rightarrow$ pressing pressure $(\mathrm{MPa})$; $y$-axis $\rightarrow$ pressing temperature $\left({ }^{\circ} \mathrm{C}\right)$; $z$-axis $\rightarrow$ briquette density $\left(\mathrm{kg} \cdot \mathrm{dm}^{-3}\right)$; colour legend $\rightarrow$ levels of material moisture: red $=5 \%$; green $=8 \%$; purple $=10 \%$; blue $=12 \%$; yellow $=15 \%$.

\subsection{Back Pressure Acting on the Pressed Plug in the Pressing Chamber}

The back pressure acting on the plug in the pressing chamber is a very important parameter during the pressing process. It prevents material from escaping the pressing chamber before pressing and facilitates pressing in the chamber. The back pressure can be attained using horizontal and vertical pressing configurations and forms as a single back pressure plug at the end of the pressing chamber with a controllable choke, friction coefficient between the briquette and the wall of the pressing chamber, shape of the pressing chamber (conicity), and the length of the crimp column. The value of this parameter is very useful to know while sizing a pressing chamber. According to the functional dependency $\rho=\mathrm{f}(\mathrm{p})$, it is possible to find this value. Based on Equation 2.32 , it is possible to calculate the value of the back pressure exerted on the plug in the 
pressing chamber. The value of the back pressure, together with the value of the radial pressure, acting over the length of the pressure chamber can be determined. The next example demonstrates the usefulness of the dependency $\rho=\mathrm{f}(\mathrm{p})$ and Equation 2.32 for calculating the back pressure acting on the plug in the pressing chamber. The calculation is applied to the design of the experimental stand. The back pressure is calculated according to the following equation:

$$
p_{G}=p_{k} \cdot e^{-\frac{4 \cdot \lambda \cdot \mu \cdot H}{D_{k}}}
$$

where :

$p_{G}$-is the back pressure in the chamber $(\mathrm{MPa})$

$p_{k}$ - axial pressure of cylinder (MPa) - in our case pressing pressure used to make a briquette of standardized quality $p_{k}=120 \mathrm{MPa}$ (see Fig. 4.3).

$\lambda$ - primary stress ratio $\sigma_{r} / \sigma_{m}$ (see Fig. 2.46). For disperse materials it is: $0<\lambda<1$.

$\mu$ - friction coefficient between the pressed material (wood) and the wall of the chamber (steel). The coefficient is $\mu=0.35$.

$H$ - length of the plug $(\mathrm{mm})$

$D_{k}$ - diameter of the pressing chamber $(\mathrm{mm})$. In our case $\mathrm{D}_{\mathrm{k}}=20 \mathrm{~mm}$.

For calculating the length of the plug. we will start from the dimensions of the pressing chamber and from the compression ratio. An example of the calculation is shown in Fig. 6.3.

The dimensions of the pressing chamber are $D_{k}=20 \mathrm{~mm}$ and $L_{k 1}=140 \mathrm{~mm}$. The volume of the pressing chamber before the first compression is $V_{k 1}=43982 \mathrm{~mm}^{3}$. As mentioned before, calculating the length of the plug uses the compression ratio for wood. This ratio compares the volume before and after compression. Using the application software and Fig. 4.3, we know that the density of a briquette pressed at a pressure of $120 \mathrm{MPa}$ and a temperature of $105^{\circ} \mathrm{C}$ was $1.12152 \mathrm{~kg}$. dm ${ }^{-3}$. The bulk density of the sawdust is around $0.140 \mathrm{~kg} . \mathrm{dm}^{-3}$. This means that the compression ratio in our case is $1.12152 / 0.140 \cong 8$. Then, the volume of the plug is $V_{k 1} / 8=5497 \mathrm{~mm}^{3}$. Since the diameter of the plug does not change, through the use of a basic formula for calculating the volume of a cylinder, the length of the plug can be calculated as $H_{1}=17.5 \mathrm{~mm}$. For calculating the second plug, we need to correct for the height of the pressing chamber. Since, in our case, we are not dealing with an interim process of pressing, the chamber is always closed. The first plug is still in the pressing chamber, and its dimension decreases the volume of the pressing chamber. By subtracting $H_{1}$ from the initial length of the chamber $L_{k 1}$, we obtain $L_{k 2}=122.5 \mathrm{~mm}$. The procedure is repeated for calculating the rest of $H_{i}$. Table 6.1 lists all calculated values for back pressure. Subsequently, we can then calculate the value of the back pressure in relation to the pressed column. 


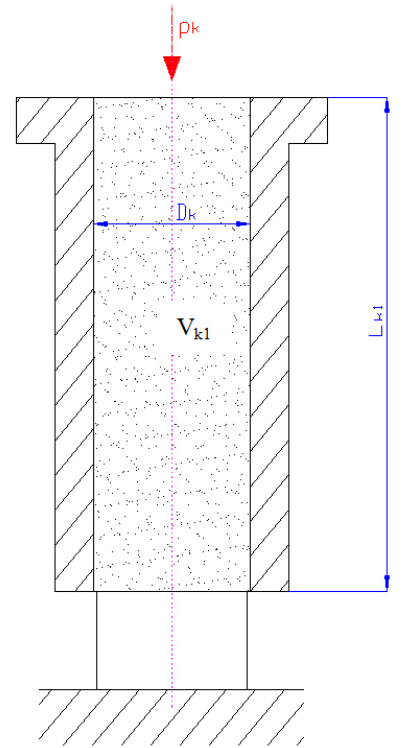

a.)

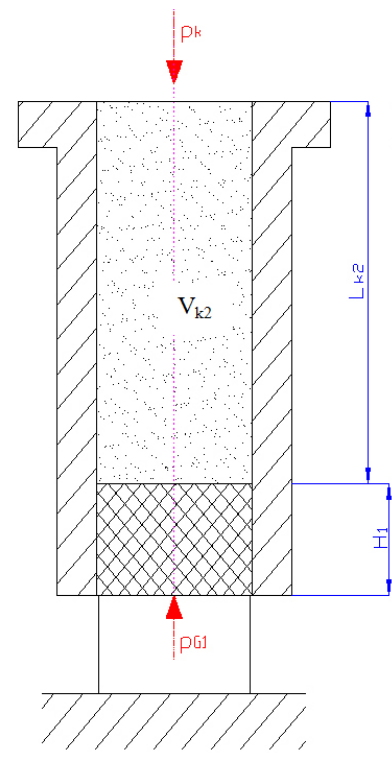

b.)

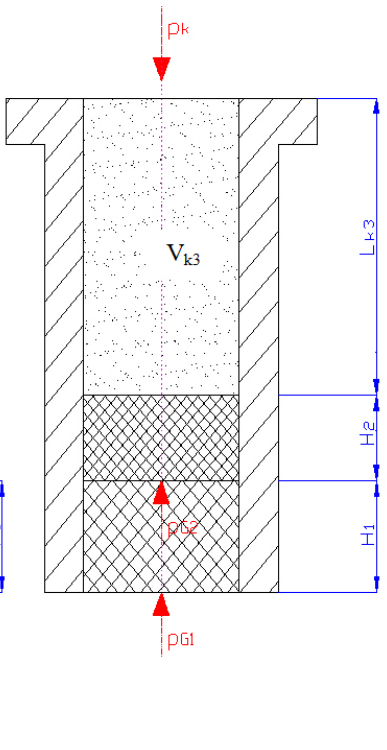

c.)

Figure 6.3: An example for calculating the length of the pressed plug (at each pressing stage) a.) filling of the chamber; b.) pressing the first plug + filling the chamber; c.) pressing the second plug + filling the chamber.

Table 6.1: Table of calculated parameters for the back pressure.

\begin{tabular}{lllllllll}
\hline $\mathbf{i}$ & $\mathbf{1}$ & $\mathbf{2}$ & $\mathbf{3}$ & $\mathbf{4}$ & $\mathbf{5}$ & $\mathbf{6}$ & $\mathbf{7}$ & $\mathbf{8}$ \\
\hline $\mathrm{L}_{\mathrm{ki}}(\mathrm{mm})$ & 140 & 122.5 & 107.19 & 93.79 & 82.07 & 71.81 & 62.83 & 54.98 \\
$\mathbf{H}_{\mathrm{i}}(\mathrm{mm})$ & 17.5 & 15.31 & 13.40 & 11.72 & 10.26 & 8.98 & 7.85 & 6.87 \\
$\mathbf{p}_{\mathrm{Gi}}(\mathrm{MPa})$ & 39.84 & 45.74 & 51.59 & 57.35 & 62.87 & 68.15 & 73.18 & 77.84 \\
\hline
\end{tabular}

As we can see from the previous table, the back pressure value increases with decreased plug length. In a closed pressing system, the pressed plug is shorter after each pressing because after each pressing, the volume of the pressing chamber decreases.

Based on this calculation, we can assume that the back pressure value will decrease with increasing length of the pressed column. Since Equation 2.32 allows for the calculation of back pressure depending on the length of the pressed column, it is possible to apply these results for the continuous pressing process. The explanation can be visualized by Figure 6.4. The principle of continuous pressing is different, meaning that the length of the pressed plug will be the same after each pressing, 
whereas earlier plugs are shifted toward the exit of the chamber. The calculations consider the parameters of the experimental stand but with the assumption that the pressing process is continuous. Figure 6.4 describes situations and effects of the backpressure during continuous pressing.

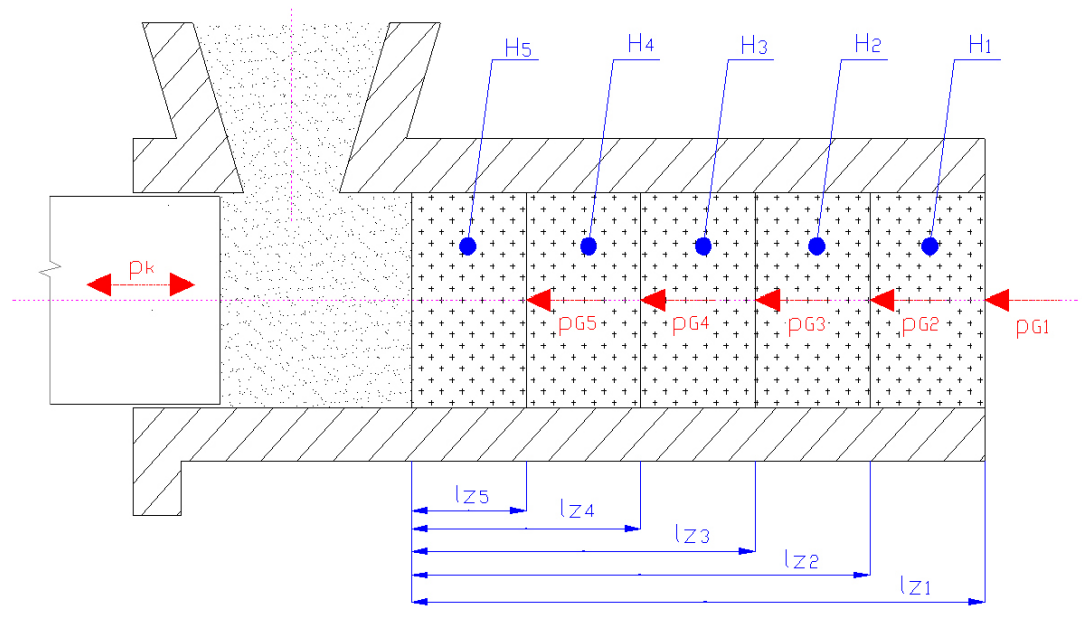

Figure 6.4: Back pressure in the continuous pressing process.

Table 6.2: Table of calculated values for the back pressure in continuous pressing process.

\begin{tabular}{llllll}
\hline $\mathbf{i}$ & $\mathbf{1}$ & $\mathbf{2}$ & $\mathbf{3}$ & $\mathbf{4}$ & $\mathbf{5}$ \\
\hline $\mathbf{l}_{\mathrm{zi}}(\mathrm{mm})$ & 87.5 & 70 & 52.5 & 35 & 17.5 \\
$\mathbf{p}_{\mathrm{Gi}}(\mathrm{MPa})$ & 0.48 & 1.45 & 4.39 & 13.23 & 39.84 \\
\hline
\end{tabular}

Based on the calculated values, we observe that the back pressure decreases as the pressed column length increases (increased length of the pressing chamber). Obviously, further experiments are necessary to verify this observation. Furthermore, it is necessary to find the optimal length of the pressing chamber depending on the pressing pressure when the desired density is achieved. This will depend also on the radial pressure. The radial pressure is possible to calculate based on the ratio between primary stresses $\lambda$ (radial/axial). It would be very useful if experiments determining the radial pressure were performed.

Figure 6.5 shows the dependence of the back pressure acting on the plug within the pressing chamber on the length of the pressed column. This dependence was defined from the calculated results by means of Equation 2.32 and their interpretation 
using figure 6.4. To define the dependence between the pressure on the overall length of the pressing chamber, it is necessary to understand, according to Equation 2.37, the values of residual radial pressure $p_{r, r}$ and material constant $A$, which gives the degree of elastic properties within the pressed material. However, these values are found only through experiments.

As above, the calculation of the backpressure uses Figure 4.3 to define the pressure at $120 \mathrm{MPa}$ and at a temperature of $105^{\circ} \mathrm{C}$. In my case, $35 \mathrm{~mm}$ briquettes were pressed which, based on the calculated parameters, should return a backpressure of approximately 13.23 MPa. This would mean that, at the values of backpressure, it should be possible to press a briquette with a density reflecting standards.

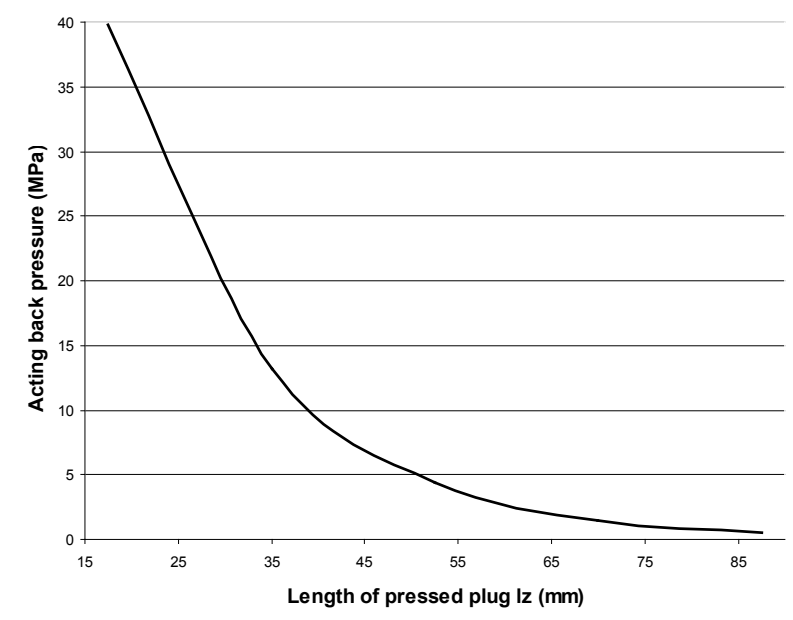

Figure 6.5: Dependence of the back pressure acting on the plug in the pressing chamber on the length of the pressed column.

\subsection{Radial Pressure Acting in the Pressing Chamber}

The radial pressure, pressing pressure, temperature, and back pressure work together in the densification process. The magnitude of acting pressure $p_{r}$ is a factor that determines whether the briquette may exit the chamber or not. If the radial pressure acting on the surface of the plug after compressing is large, the briquette could crumble after exiting the chamber due to residual radial stresses. It is therefore necessary to keep the briquette within the chamber until the residual radial stresses decrease below the strength value of the briquette. For designers, it is very interesting to know the dependence of radial pressure on the overall length of the pressing chamber; on the other hand, such information is very difficult to obtain. It is possible to calculate them; however, without the previous calculation for back pressure, it would not be possible. 
When pressing bulk materials, we are usually talking about anisotropic pressure (higher pressures are in the perpendicular direction). Equation 2.30 represents the ratio of primary stresses (radial $\sigma_{r} /$ axial $\sigma_{m}$ ). From before, we know how to calculate $p_{G}$ separately for each pressed plug. If, according to Figure 2.46 we define $p_{G}=p_{m}$, we should be able to determine the value of radial pressure using the following relations for any point in the pressing chamber.

$$
\lambda=\frac{\sigma_{r}}{\sigma_{m}}=\frac{p_{r}}{p_{m}}=\frac{p_{r}}{p_{G}} \Rightarrow p_{r}=\lambda p_{G}
$$

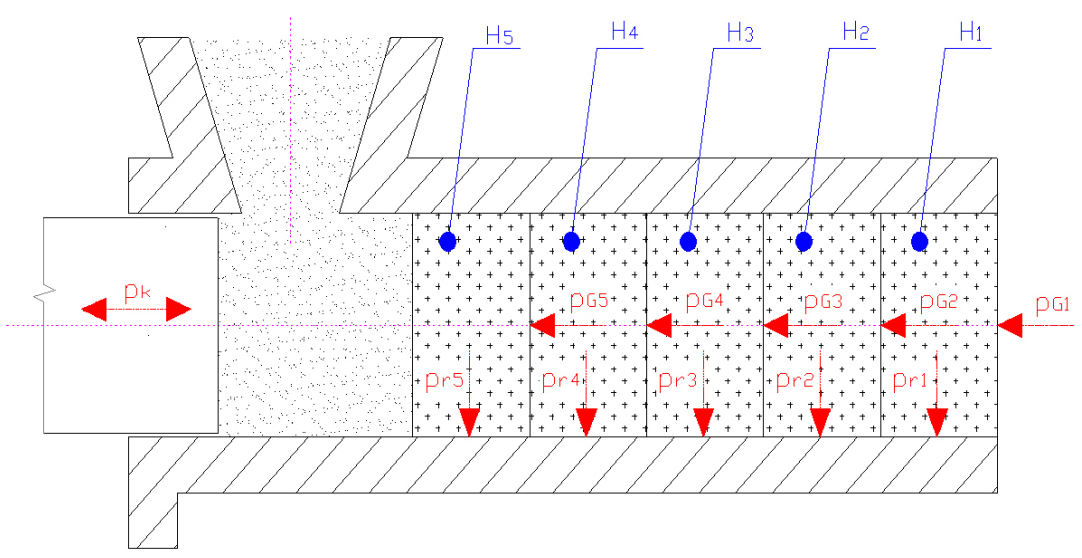

Figure 6.6: Behaviour of radial pressure during continuous pressing.

Since $0<\lambda<1$, we set $\lambda=0.9$. Then, from the values of $p_{G i}$ in Tab. 6.2, we calculate the values for $p_{r i}$ :

Table 6.3: Table of the calculated radial pressures.

\begin{tabular}{llllll}
\hline $\mathbf{i}$ & $\mathbf{1}$ & $\mathbf{2}$ & $\mathbf{3}$ & $\mathbf{4}$ & $\mathbf{5}$ \\
\hline $\mathbf{p}_{\mathrm{mi}}=\mathbf{p}_{\mathrm{Gi}}(\mathrm{MPa})$ & 0.48 & 1.45 & 4.39 & 13.23 & 39.84 \\
$\mathbf{p}_{\mathrm{ri}}(\mathrm{MPa})$ & 0.43 & 1.31 & 3.95 & 11.91 & 35.86 \\
\hline
\end{tabular}


Based on the calculated values, we can see that the radial pressures decrease with increasing length of the pressing chamber.

\subsection{Length of the Pressing Opening - Pressing Chamber}

If we know the value of axial pressure acting on the plug for which the briquette will not crumble when it leaves the chamber, based on Equation 2.45, we can calculate the optimal length of the pressing opening (area in the pressing chamber where the given pressing pressure is acting on the plug). After modification of Equation 2.45, we get:

$$
L_{k}=\frac{\ln \frac{p_{m}}{p_{m 0}} \cdot r}{2 \cdot \mu \cdot G \cdot \nu_{R L}}
$$

where:

$p_{m}$ - pressing pressure on the plug (MPa);

$p_{m o}$ - initial pressing pressure (MPa); in our case, the pressing pressure which gives the briquette at a quality defined by standards $p_{k}=120 \mathrm{MPa}$ (see Fig. 4.3).

$r$ - radius of the pressing chamber $(\mathrm{mm})$; in our case $r=10 \mathrm{~mm}$.

$\mu$ - friction coefficient between the pressed material (wood) and the material of the chamber (steel); coefficient of friction between wood/steel is $\mu=0.35$.

$G$ - ratio of the elasticity module for lateral and radial directions in the fibre bundles $\left(E_{L} / E_{R}\right)$; for pine sawdust $E_{R}=320 \mathrm{MPa} ; E_{L}=6634 \mathrm{MPa}$.

$v_{R L}$ - Poisson's constant; for pine sawdust $v_{R L}=0.016$.

Based on the calculated results in Table 6.2 and the conclusions described by the assumption that when applying a pressure $p_{m}=13.23 \mathrm{MPa}$ on the plug, the briquette should not crumble upon exiting the chamber, this value on the plug will be considered when calculating the length of the press opening $L_{k}$. If we substitute the defined values into Equation 6.2, the calculated length of the press opening is $L_{k}=$ $94.97 \mathrm{~mm}$. In this way, we can calculate the length of the pressing chamber for the previously calculated values of $p_{m}$. It is necessary, though, to remind the reader that it is very important to interpret the results appropriately. Improper interpretation can result in a very large error in sizing the pressing chamber. This means that calculated values for $L_{k}$ are values representing the length of the pressing chamber. However, they represent areas of the pressing chamber which produce pressure on the plug. For simplicity, an illustration is given in Figure 6.7, which is based on Figure 2.47. 
Table 6.4: Table of the calculated pressing chamber lengths.

\begin{tabular}{llllll}
\hline $\mathbf{I}$ & $\mathbf{1}$ & $\mathbf{2}$ & $\mathbf{3}$ & $\mathbf{4}$ & $\mathbf{5}$ \\
\hline $\mathbf{P}_{\mathrm{mi}}=\mathbf{p}_{\mathrm{Gi}}(\mathrm{MPa})$ & 0.48 & 1.45 & 4.39 & 13.23 & 39.84 \\
$\mathbf{L}_{\mathrm{k}}(\mathrm{mm})$ & 237.80 & 190.19 & 142.48 & 94.97 & 47.49 \\
\hline
\end{tabular}

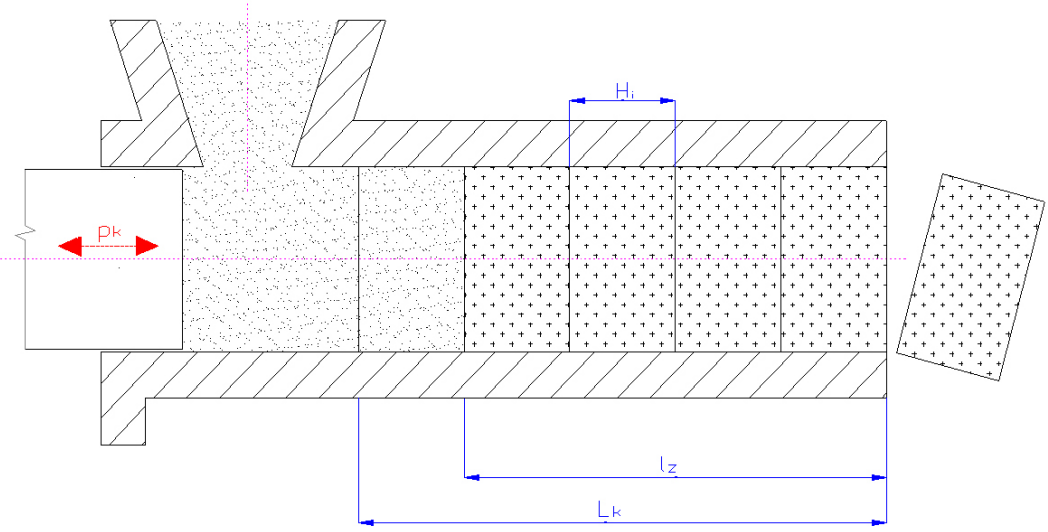

Figure 6.7: Calculation of pressing chamber length. 